\title{
Zu Ende erzählen: Leben und Sterben im Text
}

Franziska Gygax

Fast wie Scheherazade, die durch ihr Erzählen den Tod abwenden konnte, finden wir in zahlreichen sogenannten Autothanatografien ${ }^{1}$ todkranke ErzählerInnen, die dem Tod entgegen schreiben und auf diese narrative Weise ihrem Lebensende Kontinuität verleihen. Obwohl ihr Narrativ mit ihrem Tod enden wird, sind diese Geschichten einerseits durch ihren Fokus auf das (gelebte) Leben charakterisiert, und andererseits leben sie durch unsere Lektüre und Rezeption weiter.

Obwohl der Tod den AutorInnen, deren Texte ich besprechen werde, gewiss und auch omnipräsent ist, stellt er keine Instanz dar, die eindeutig im Text eingeschrieben ist. Michel de Certeau, der sich vor allem mit der Theorie der Alltagspraxis befasste, zu der letztlich auch das Sterben und der Tod gehören, hält fest, »dass der Tod nicht benennbar ist. Aber er schreibt sich in den Diskurs des Lebens ein, ohne dass es möglich wäre, ihm einen bestimmten Platz zuzuweisen. « Für de Certeau ist der Tod nicht beschreibbar, jedoch weist er ihm eine Präsenz zu, die unabdingbar mit dem Leben verbunden ist. Das Kapitel, in dem er diese Aussagen festhält, ist überschrieben mit Sterben: Das Unsagbare (335). Für de Certeau sind also das Sterben wie der Tod unsagbar, und er weist auf die Tabuisierung des Todes in unserer Gesellschaft hin: "[E]r [der Sterbende] wird zensiert, der Sprache beraubt und mit dem Leichentuch des Schweigens zugedeckt: das Unsagbare.« (336) Er, der Philosoph aber, der über den Sterbenden schreibt, macht sich ein Bild von ihm und erschafft so durch Sprache, was eigentlich nicht benennbar ist: "Warum soll man nicht im Namen eines unmöglichen Sprechens schreiben?« (342) Diese Feststellung führt de Certeau zum Schriftsteller, der für ihn eine Art Sterbender ist, der zu sprechen versucht, um "mit dem Wortschatz des Vergänglichen Sätze zu produzieren« (346), die von LeserInnen rezipiert werden. Der/die Schreibende sieht sich immer mit dem Verlust konfrontiert, der sich aus der Inkongruenz zwischen Zeichen und Bedeutung ergibt. Der Text wird vom "Anderen" (der Leserschaft) aufgenommen, dessen Antwort

1 Ich übernehme diesen Begriff aus der anglophonen Autobiografieforschung, wo er schon länger verwendet wird.

2 Michel de Certeau, Kunst des Handelns. Aus dem Frz. von Ronald Voullié, Berlin 1988,346 . 
von dem/der Schreibenden losgelöst (verloren) ist. Dieser Vorgang ist dann bezeichnenderweise mit dem Tod verbunden, denn durch die Sätze auf dem jetzt beschriebenen Blatt wird der Tod eingeschrieben; das nicht mehr weisse, leere Blatt ist vollgeschrieben und hat den geschützten Bereich verlassen und befindet sich "schon fast im Raume des Todes" (347).

Was bedeutet nun aber die Analogie zwischen Schreibendem und Sterbendem, wenn der Schreibende selbst ein "tatsächlich" Sterbender ist und sich mit dem baldigen Sterben und Tod konfrontiert sieht? Wie werden das Sterben und der Tod in autobiografischen Texten von den Sterbenden beschrieben? Charakterisiert de Certeaus "unmögliches Sprechen" ihre Texte, oder erfahren wir etwas über die Grenzerfahrungen zwischen Leben und Tod?

In den drei ausgewählten autobiografischen Texten von Jenny Diski, In Gratitude (2016), Christoph Schlingensief, So schön wie hier kanns im Himmel gar nicht sein! Tagebuch einer Krebserkrankung (2009), und Harold Brodkey, This Wild Darkness: The Story of My Death (1996), die alle über ihr Sterben und ihren bevorstehenden Tod schrieben, soll der Frage nachgegangen werden, wie denn dieses "Unsagbare» repräsentiert wird und welche narrative Eigenheiten auszumachen sind. Ich habe diese drei AutorInnen ausgewählt, weil sie die Problematik des Diskurses explizit thematisieren und mit ihren eigenen Erfahrungen erhellen. Alle drei AutorInnen erwähnen ihre "natürliche "Reaktion angesichts einer todbringenden Krankheit, die das Nachdenken, Sprechen und Schreiben darüber verlange, denn das sei schliesslich ihr Beruf und ihre Vokation. Auch zahlreiche andere Autobiografen, die über ihren bevorstehenden Tod schreiben, setzen sich damit auseinander, schreibend zu erforschen, was letztlich niemand von uns wissen kann.

Die britische Schriftstellerin Jenny Diski erkrankte 2014 an Lungenkrebs und starb im April dieses Jahres. Sie verfasste monatliche Kolumnen über ihre Krankheit in der London Review of Books und beschrieb offen, jedoch ganz unsentimental die damit verbundenen Schmerzen, Ängste, schmerzhaften Erinnerungen an die Vergangenheit, aber auch an positive Ereignisse. Im Buch In Gratitude wurden diese Einträge nun gesammelt herausgegeben (jeweils ohne Datumsangabe wie in der Zeitschrift). Wie die meisten Krankheitserzählungen beginnt auch Diski mit der Diagnose, die sie öffentlich machen will, aber gleichzeitig kritisiert sie die Reaktion der Leserschaft auf solche öffentlich gemachten Diagnosen: 
"It's a delicate balance, this publicising of one's cancer. The public's interest is fixated on when each of them will die. For some reason cancer is the disease of choice for public tonguewagging. It has that something, that je ne sais quoi, not just death, but how long known beforehand: how will she die, should she choose to try for a longer life by accepting treatment, or settle for palliative care which at its best is a comfortable death without pain. $\ll^{3}$

Nebst Diskis Seitenhieb auf einen voyeuristischen Zug der Leserschaft nennt sie ihren Beruf als Schriftstellerin die treibende Kraft hinter ihrem öffentlichen Krebstagebuch, das sie kurz nach ihrer Diagnose zu verfassen beginnt:

")Well, I suppose I'm going to write a cancer diary.< [ ...] I'm a writer, have been since I was small, and have earned my living at it for thirty years. I write fiction and non-fiction, but it's almost always personal.«(Pos. 135)

Die natürliche Reaktion des schriftlichen Offenlegens ihrer Krankheit mit all ihren Ängsten und einhergehenden Komplikationen und Schmerzen betrifft auch immer die Frage nach dem Sterben und dem Tod, und im Angesicht der tödlichen Krankheit werden sie, wie Diski meint, neu beschrieben, denn bis anhin habe sie sicher öfters über den Tod sinniert, aber nie in diesem absoluten Sinn. Und jetzt, da diese Momente bald Realität werden können, findet sie die Sprache nicht dafür:

"The prospect of extinction comes at last with an admission of the horror of being unable to imagine or be part of it, because it is beyond the you that has the capacity to think about it. I learned the meaning of being lost for words; I came up against the horizon of language.«(Pos. 1779)

Die Einsicht, dass dem Sterben und dem Tod die Sprache versagt bleibt - oder umgekehrt formuliert: der Sprache bleibt das Sterben und der Tod versagt -, finden wir auch bei Schlingensief, dem Theaterregisseur und Autor, der 2008 an einem Lungentumor erkrankte und 2010 daran starb. Im Vorwort nennt er sein Buch eine Schrift "gegen die Sprachlosigkeit des Sterbens « ${ }^{4}$, und er ist überzeugt, dass

\footnotetext{
3 Jenny Diski, In Gratitude, London 2016. Kindle DX Version. Von Amazon.de. abgerufen; Pos. 1975-1980. Alle Nachweise beziehen sich auf diese Ausgabe.

4 Christoph Schlingensief, So schön wie hier kanns im Himmel gar nicht sein! Köln 2009, 9. Alle weiteren Verweise im Text beziehen sich auf diese Ausgabe.
} 
ihm gerade das Schreiben über und das Erzählen von seiner Erkrankung eine grosse Hilfe bei der Auseinandersetzung mit seiner Krebserkrankung bot. Die narrative Kraft, wie ich die Wirkung des Erzählens der Sterbenskranken nennen will, wird von ihm als eine Distanzierung beschrieben, die Möglichkeit, die "Erkrankung vor sich zu stellen und sich selbst von aussen zu betrachten - dieser ganzheitliche Blick ist so wichtig und hilfreich" (9f.). Obwohl Schlingensief sich hier nicht ausschliesslich auf das Schreiben bezieht, sondern generell die Wichtigkeit des Erzählens und Mitteilens der Erkrankten betont, wird die Wirkung des Narrativen einsichtig.

Bei Harold Brodkey, dem amerikanischen Schriftsteller, der 1996 an den Folgen seiner AIDS-Erkrankung starb, finden wir eine explizitere Beschreibung von dem, was für ihn Tod ausmacht. Obwohl zuerst als Frage formuliert und letztlich als klar imaginiert bezeichnet ("Or this is what I imagine, on the approach $\aleph^{5}$ ) tönt seine Beschreibung erstaunlich präzise und analytisch, wenn auch sehr abstrakt:

"Is death other than silence and nothingness? [...] Death itself is soft, softly lit, vastly dark. The self becomes taut with metamorphosis and $[\ldots]$ to have a not-quite-great-enough fearlessness toward that immensity of the end of individuality, toward one's absorption into the dance of particles and inaudibility. [...] This one [the sense of real death] is a stillness and represents a sifting out of identity and its stories, a breaking off or removal of the self, and a devolution into mere effect and memory, outspread and not tightly bound $[\ldots] . \lll(23 \mathrm{f}$.)

Der Tod scheint etwas Beruhigendes, Sanftes auszustrahlen, da das Subjekt sich langsam aufzulösen beginnt und in unendlich vielen Teilen sich der Individualität entzieht. Gleichzeitig geht mit dieser Metamorphose, wie Brodkey den "Übergang" nennt, eine Beruhigung und Ruhe einher, die diese Auflösung des Selbst umgeben. Diese stoische und unsentimentale Annäherung an den Tod zeichnet auch Brodkeys spätere Aufzeichnungen aus, wenn er sogar eine Art Glücksein mit dem Tod assoziiert: "The world still seems far away. And I hear each whisper as it slides along. And yet I am happy - even overexcited, quite foolish. But happy. It seems very strange to think one could enjoy one's death." (176) Diese Feststellung steht fast am Ende von Brodkeys Ausfuihrungen und ist auch charakteristisch für den tatsächlichen Schluss seines Buches - oder Lebens: »It [genuine amazement] is all around me." (177)

5 Harold Brodkey, This Wild Darkness:The Story of My Death, New York 1996, 23 f. Alle Angaben im Text entstammen dieser Ausgabe. 
Wir wissen nicht, wie Brodkey sich während der letzten Momente seines Lebens fühlte, aber wir lesen die mit offensichtlich ruhiger Gewissheit geschriebenen Zeilen eines Sterbenden und nehmen so teil an seinem Ende, aber mit der Beruhigung, dass dieser Text lebensbejahend ist. Auch Schlingensiefs Tagebuch endet mit der Aufforderung an das Leben, auch wenn ihm und uns klar ist, dass das "Weihnachtswunder" (252) keine Heilung bringen kann. Er spricht ebenfalls vom Glück, aber gleichzeitig auch vom Zweifel, der damit einhergeht, jedoch liegt der Fokus auf dem Leben:

»Und dieses Leben, sei es noch so kurz, beinhaltet den Zweifel und das Glück, das Wissen und das Unwissen. Und es ist nichts Fatalistisches und nichts Peinliches, es ist auch nichts Niederträchtiges oder Berechnendes - es ist einfach ein ganzes Leben." (255)

Während Brodkeys und Schlingensiefs letzte Einträge sich wieder ganz dem Leben zuwenden, ist Diskis Text verschlüsselter und muss im Zusammenhang mit der sehr belasteten Beziehung zu ihrer Mutter gelesen werden. Der letzte Abschnitt ihres Textes ist ein Konglomerat aus schmerzhafter Erinnerung an ihre Mutter und deren unverständliche Reaktion auf Diskis erste Menstruation und eigenen Gedanken über Krankheit, die sie mit ihrer ersten Erfahrung als menstruierendes Mädchen assoziiert: „Unwell, after all, is not ill" (3161), wobei "ill« für (ihre) Krankheit steht und "unwell» für die Menstruation (wie ihre Mutter es nannte). Zu dieser Vermischung hinzu kommt dann der Verweis auf eine Szene aus Louisa May Alcotts Little Women mit Diskis Bemerkung, dass dies alles zusammen eine viel bessere Geschichte sei als Disneys Beauty and the Beast (vgl. 3166). Anders als Brodkey und Schlingensief lässt Diski ihren Assoziationen freien Lauf und kreiert eine sprachlich wundervolle Gedanken- und Erinnerungswelt für sich und die LeserInnen. Auch ihr Text endet nicht mit einer Auseinandersetzung mit dem Sterben oder dem Tod, sondern verweist und zählt auf die kreative Imagination.

Wie eingangs erwähnt, befassen sich die meisten Autothanatografien mit dem Leben und viel weniger mit dem Sterben und dem Tod. Ivan Callus spricht von "einem Skript der Lebenden", das diese Autothanatografien ausmache. ${ }^{6}$ Dieses Skript ist von einer Intensität gekennzeichnet gerade eben durch dieses »letzte« Erzählen bestimm-

${ }^{6}$ Ivan Callus, (Auto)Thanatography or (Auto)Thanatology?: Mark C. Taylor, Simon Critchley and the Writing of the Dead, Forum of Modern Language Studies 41, 4 (2005), 427-438, hier 437 (Übersetzung der Autorin). 
ter Lebenserfahrungen. Susan Bainbrigge erklärt dieses Verhältnis zwischen Leben und Tod in ihrer Einleitung zu einem Sonderband zu Autothanatografien: "[W] hen thanatos replaces the bios in autobiography it most often highlights the intensity of the experiences being described. " ${ }^{7}$ Ist es allein die Gewissheit, bald sterben zu müssen, welche diese Intensität der erzählten Erfahrungen hervorruft, und/oder das Wissen darum, diese Lebenserfahrungen endgültig hinter sich zu lassen? Und inwieweit beeinflusst der Verlauf der Krankheit das Erinnern und Niederschreiben? Letztlich können wir keine eindeutigen Antworten finden, jedoch können wir bestimmte gemeinsame Merkmale dieser Narrationen beobachten.

Alle drei AutorInnen beginnen ihre Aufzeichnungen mit ihrer Krankheitsdiagnose, die sie gänzlich unvorbereitet trifft, wobei Diski ihr erstes Kapitel explizit mit "Diagnosis « überschreibt. Diese Mitteilung birgt in sich schon eine zu erwartende Dringlichkeit und Verunsicherung, wobei die LeserInnen gleichzeitig auch auf eventuelle schwierige Passagen vorbereitet werden können, die Schmerzen und Leiden beschreiben. Die schwere Krankheitsdiagnose ohne Hoffnung auf Heilung zu Beginn eines autobiografischen Texts markiert dessen zeitlich begrenzten narrativen Charakter: Solange die Autobiograflnnen am Schreiben sind, sind sie auch am Leben, und somit begleitet die Leserin die sterbende Person im Schreiben wie im Leben, da beide unumgänglich miteinander verbunden sind. Jedoch ist es der tatsächliche Tod, welcher der Autobiografin in unseren Beispielen die Autor-ität verleiht und nicht der abstrakte Tod, wie Walter Benjamin es ausdrückt: »Der Tod ist die Sanktion von allem, was der Erzähler berichten kann. Vom Tod hat er seine Autorität geliehen. $\aleph^{8}$ Der Tod, von dem Benjamin im Zusammenhang mit dem Erzählen spricht, meint das Ende der Erzählung, auf das hin wir immer lesen und in dessen Erwartung wir uns bewegen. Lesen wir einen Text mit dem Wissen, dass sein Ende mit dem Lebensende des Autors zusammenfällt, scheint das Ende des Textes schon vorweggenommen. Aber da die von Benjamin genannte Autorität des Erzählers in einem solchen Text omnipräsent ist, wird unser Lesen kein antizipierendes, sondern ein emphatisches, das diese "Autorität" gänzlich akzeptieren kann/muss.

\footnotetext{
7 Susan Bainbrigge, Introduction, in: Autothanatography (Special Issue), Forum of Modern Language Studies 41, 4 (2005), 359-364, hier 363f.

8 Walter Benjamin, Der Erzähler. Betrachtungen zum Werk Nikolai Lesskows, in: Gesammelte Schriften, Bd. II, 2, hg. v. RolfTiedemann/Hermann Schweppenhäuser, Frankfurt a.M. 1977, 450.
} 
Wie aber gehen Brodkey, Schlingensief und Diski mit ihrer unheilbaren Krankheit um, ihrem Leiden und der Gewissheit, bald sterben zu müssen? Was und wie erzählen sie nebst ihrer Auseinandersetzung mit dem Tod an sich? Was tritt an die Stelle des Sterbens und des Todes, wenn sie nicht in Sprache gefasst werden können und doch omnipräsent sind wie bei Diski?

Alle drei schauen zurück auf ihr Leben und auf ihre Arbeit, wobei aber Schlingensief vermehrt auf die Gegenwart fokussiert und gleichzeitig an eine Zukunft glauben will. Diskis Zurückschauen bedeutet vor allem eine schmerzhafte Auseinandersetzung mit Doris Lessing, der berühmten Schriftstellerin, die Diski als Teenager in ihr Haus aufnahm. Erst jetzt ist es ihr möglich, über die komplizierte und belastete Beziehung zu schreiben, nicht nur, weil Lessing tot ist, sondern weil ein tiefes Bedürfnis sie dazu dränge, darüber vor ihrem eigenen Tod das "letzte Wort «" zu schreiben. Es wird klar, dass die Aufarbeitung dieser Beziehung gleichzeitig bedeutet zurückzuschauen auf ihre eigenen Eltern, die Diski kein Heim und keinen Schutz bieten konnten. Dieser lange erste Teil der Autothanatografie erwähnt die Krankheit nicht mehr bis zu Beginn des zweiten Teils, der mit Chemo and Me überschrieben ist und hauptsächlich der Chemotherapie und ihren Nebenwirkungen gewidmet ist, die mit der Schriftstellerin eigenen Ironie immer wieder auch distanziert und ohne Sentimentalität betrachtet werden wie zum Beispiel in der folgenden Aussage:

"There was a stasis in the other, non-cancer-life. [...] I tell myself it's because I'm not so much in charge in cancer-world. I don't think I've ever felt so not in charge. This is one of the surprises of being cancered.«(1456-1462)

Bei Schlingensief steht die Krebserkrankung stärker im Mittelpunkt, und er beschreibt ausführlich seinen Krankenhausaufenthalt und die darauffolgende Chemotherapie, jedoch viel weniger distanziert als Diski und Brodkey. Die doch viel stärker wahrnehmbare Unmittelbarkeit ist wohl deshalb spürbar, weil er seine Aufzeichnungen direkt in ein Mikrofon spricht. Auch er schaut zurück und berichtet über die nicht unbelastete Beziehung zu seinen Eltern, so wie auch Brodkey von seinen Eltern erzählt, wobei die traumatischen Erfahrungen des sexuellen Missbrauchs durch den Stiefvater im Zentrum

9 In einem Interview erwähnt Diski, dass nicht Lessing das letzte Wort haben sollte: "So I thought I would get my own last word in before I pop off." https://www. theguardian.com/books/2014/dec/07/jenny-diski-doris-lessing-cuckoo-in-nest (21.6.2016). 
stehen. Die Beschäftigung mit der eigenen Familie findet in allen drei Texten statt; eine rückblickende Auseinandersetzung mit ausserordentlich schwierigen Familienverhältnissen erscheinen bei Diski und Brodkey nicht ganz unerwartet, denn eine Aufarbeitung von bisher eventuell Verdrängtem wird sichtbar, das vor dem unmittelbar bevorstehenden Lebensende betrachtet werden soll.

Nebst dem Rückblick auf schmerzhafte Erfahrungen in ihrem Leben und Beschreibungen zunehmender Schmerzen und körperlicher Behinderungen schreiben alle drei AutorInnen von ihrer Arbeit, die nebst den unterstützenden Lebenspartnern den wichtigsten Platz einnimmt. Für Diski und Brodkey, die Schriftsteller, ist das Schreiben lebensnotwendig, und auch für Schlingensief, der seine Gedanken ja ins Mikrofon spricht, bietet diese Ausdrucksmöglichkeit Hilfe, sich "gegen den Verlust [s]einer Autonomie zu wehren" (9). So ist das Schreiben/Erzählen für die sterbenden Autoren lebensnotwendig, obwohl es den Tod mitdenkt oder sogar thematisiert. In diesem Fall heisst »zu Ende erzählen« denn auch nicht, dass der Text mit dem Tod des/der Schreibenden endet, sondern dass dieser Text gerade durch die Allgegenwart des Todes lebt und von den LeserInnen als solcher aufgenommen wird. Der/die Sterbende und Schreibende hat mit dem »Wortschatz des Vergänglichen" Sätze produziert, die uns ihrem Tod näher bringen, weil sie direkt vom Ende ihres Lebens kommen: Ihr Ende kann unser Anfang zum Unsagbaren sein.

— Franziska Gygax ist Titularprofessorin am Englischen Seminar der Universität Basel. $\mathrm{Zu}$ ihren Arbeitsgebieten gehören insbesondere die Autobiografieforschung, Gertrude Stein-Forschung, das Gebiet Literatur und Medizin sowie die Medical Humanities. 